\title{
DeuZikaChico: o poder da AGI no monitoramento e combate de epidemias como a de Dengue, Zika e Chikungunya
}

\author{
Alternative Title: DeuZikaChico: the power of AGI to monitor and combat \\ epidemics such as Dengue, Zika and Chikungunya
}

\author{
Luiz Henrique de Andrade ${ }^{1}$, Brunna de Sousa Pereira Amorim ${ }^{1}$, Maxwell Guimarães de \\ Oliveira $^{1}$, André Luiz Firmino Alves ${ }^{1,2}$, José Nathaniel Lacerda de Abrante ${ }^{1}$, Daniel Farias \\ Batista Leite ${ }^{1}$, Júlio Henrique Rocha ${ }^{1}$, Cláudio de Souza Baptista ${ }^{1}$ \\ ${ }^{1}$ Laboratório de Sistemas de Informação, Universidade Federal de Campina Grande (UFCG), Brazil \\ ${ }^{2}$ Coordenadoria de Tecnologia da Informação, Universidade Estadual da Paraíba (UEPB), Brazil \\ \{luiz.andrade, brunna.amorim, jose.abrante\}@ccc.ufcg.edu.br, maxwell@ufcg.edu.br \\ andre@uepb.edu.br, \{danielfarias, juliorocha\}@copin.ufcg.edu.br, baptista@dsc.ufcg.edu.br
}

\section{RESUMO}

Com o advento da web social, usuários contribuem ativamente compartilhando seu conteúdo em vez de simplesmente navegar na Web. Esses usuários têm se tornado verdadeiros sensores humanos através de plataformas colaborativas como as LBSNs (Redes Sociais Baseadas em Localização), produzindo uma inteligência coletiva na resolução de problemas. Sensores humanos são responsáveis pela produção de VGI (Informação Geográfica Voluntária) e AGI (Informação Geográfica Ambiental), informações de muita utilidade nos mais diversos domínios de aplicação, como o das Cidades Inteligentes. Esses sensores têm auxiliado na resolução dos mais diversos problemas do espaço urbano, contribuindo para a melhoria da qualidade de vida dos cidadãos. Atualmente, o crescente aumento no número de casos de doenças transmitidas pelo mosquito Aedes aegypti, como a Dengue, Zika e a febre Chikungunya, tem levado o Brasil e muitos outros países do mundo a um estado de alerta no combate à proliferação do mosquito. O método de combate mais eficiente na atualidade depende da população tomando medidas profiláticas, representando um desafio para as autoridades locais. Neste contexto, este artigo apresenta o DeuZikaChico, um arcabouço que faz uso de tecnologias de geoprocessamento, plataformas móveis, crowdsourcing e redes sociais, com o objetivo de propiciar aos gestores públicos um melhor monitoramento de epidemias com o apoio imprescindível da sociedade.
Permission to make digital or hard copies of all or part of this work for personal or classroom use is granted without fee provided that copies are not made or distributed for profit or commercial advantage and that copies bear this notice and the full citation on the first page. To copy otherwise, to republish, to post on servers or to redistribute to lists, requires prior specific permission and/or a fee.

SBSI 2016, May 17th-20th, 2016, Florianópolis, Santa Catarina, Brazil Copyright SBC 2016.

\section{Palavras-Chave}

AGI, Combate, Epidemia, SIG, Twitter, Zika

\begin{abstract}
With the advent of the social web, users contribute actively sharing their content instead of just navigate the Web. Such users have become truly human sensors through collaborative platforms such as the LBSNs (Location-Based Social Networks), bringing a collective intelligence for problems solving. Human sensors are in charge of producing VGI (Volunteered Geographic Information) and AGI (Ambient Geographic Information), very useful information in the most diverse application domains, such as the Smart Cities. These sensors assist on solving various problems of urban areas and contributing to the citizens' life quality improvement. Currently, the growing number of diseases cases transmitted by the Aedes aegypti mosquito, such as Dengue, Zika and Chikungunya, has led both Brazil and other countries to an alert state. The most efficient combating method nowadays relies on the population taking preventive actions, posing a challenge to the local authorities. In this context, this paper presents the DeuZikaChico, a framework that makes use of GIS technologies, mobile platforms, crowdsourcing and social networking, with the purpose of providing public managers better monitoring of epidemics with the support from the society.
\end{abstract}

\section{Categories and Subject Descriptors}

H.5.3 [Information Interfaces and Presentation]: Group and Organization Interfaces-Collaborative computing

\section{General Terms}

Management, Human Factors

Keywords

AGI, Combating, Epidemic, GIS, Twitter, Zika 


\section{INTRODUÇÃO}

A Dengue é uma doença infecciosa transmitida pelo mosquito Aedes aegypti que acomete milhares de pessoas no Brasil a cada ano [12], sendo muito difícil de ser erradicada devido às condições ambientais muito favoráveis à proliferação do transmissor. Recentemente, foi descoberto que o mosquito transmissor da Dengue também é o responsável pela transmissão do Zika vírus (ZIKV) e da febre Chikungunya, despertando preocupação da população que tem sofrido com uma epidemia destas doenças.

Nos últimos meses, pesquisadores descobriram fortes indícios de relação entre o Zika vírus e o crescente número de más formações congênitas detectadas em bebês [2]. Há também suspeita de relação com a síndrome de Guillain-Barré em adultos. O número de casos de má formação em bebês começou a subir de tal forma que o governo entrou em alerta vermelho contra o Zika vírus, lançando campanhas para reforçar o combate à proliferação do mosquito transmissor, numa tentativa emergencial de evitar novas contaminações e reduzir consequentemente os casos de má-formação.

Em epidemias como a do Zika vírus, é muito importante localizar e eliminar os elementos causadores de forma rápida e imediata. Neste sentido, o uso de ferramentas computacionais desempenha um papel indispensável no auxílio dos agentes de saúde na linha de frente neste combate. Sistemas de Informação Geográficos (SIG) têm sido muito utilizados $[6,8]$, sobretudo por produzirem uma visualização intuitiva e precisa de dados espaciais.

Com o advento dos dispositivos móveis como os smartphones e tablets, somado ao desenvolvimento das redes sociais, os cidadãos têm se tornado verdadeiros sensores humanos, permitindo o uso de plataformas colaborativas. Esse é um fenômeno conhecido como Crowdsourcing [15], que envolve comunidades on-line às quais combinam os esforços de usuários que se tornam voluntários na produção de informações valiosas em diferentes domínios. Estes voluntários podem ser utilizados para produzir um entendimento detalhado da dinâmica das cidades, possibilitando a descoberta de problemas no espaço urbano [5]. Por exemplo, sensores humanos podem ser explorados para produzirem informações espaçotemporais sobre focos do mosquito Aedes aegypti ou casos de pessoas que foram diagnosticadas com o Zika vírus. Esse tipo de informação é conhecida como Informação Geográfica Ambiental (do inglês, AGI), uma variação da Informação Geográfica Voluntária (do inglês, VGI) que se vai além da geografia, representando comportamentos dos indivíduos no contexto geográfico [14].

Neste contexto, este trabalho propõe o ambiente DeuZikaChico, um SIG Web para monitoramento e combate em tempo real a epidemias, como a da Dengue, Zika e Chikungunya. $\mathrm{O}$ ambiente reúne informações espaço-temporais de forma intuitiva e eficiente, fornecidas por agentes de saúde e pela população atuando como sensores humanos. Enquanto os agentes de saúde atuam como usuários diretos do ambiente, a população tem a opção de atuar também como usuários diretos ou através da rede social Twitter ${ }^{1}$, já que o DeuZikaChico monitora, em tempo real, as mensagens relevantes da rede social e às exibe em seu mapa interativo. Um app para dispositivos móveis também foi desenvolvida no sentido de facilitar o cadastramento de denúncias de focos e visitas dos agentes.

\footnotetext{
${ }^{1}$ http://www.twitter.com/
}

A principal contribuição deste trabalho está na proposição de um arcabouço que faz uso de tecnologias de geoprocessamento, plataformas móveis, crowdsourcing e redes sociais, que juntos propiciam aos gestores públicos melhor monitoramento de epidemias, com o apoio imprescindível da sociedade. Também, o arcabouço possibilita aos cidadãos uma fiscalização das ações de governo no combate ao mosquito, considerando aspectos espaciais e temporais. Não foram encontradas soluções equivalentes abrangendo todos estes aspectos abordados neste trabalho de forma integrada num único arcabouço computacional.

O restante do artigo está estruturado da seguinte maneira: na seção 2 são discutidos os trabalhos relacionados encontrados na literatura; na seção 3, apresenta-se o arcabouço proposto em detalhes; na seção 4 , descreve-se um estudo de caso demonstrando a utilidade da solução proposta; por fim, a seção 5 conclui este artigo com discussões sobre trabalhos futuros.

\section{TRABALHOS RELACIONADOS}

VGI surgiu nos últimos anos como uma fonte de dados espaciais poderosa e alternativa, consistindo de dados geográficos obtidos voluntariamente por indivíduos que atuam como sensores [9]. Um dos projetos mais representativos e também conhecidos de VGI é o OpenStreetMap (OSM) [10], que reúne voluntários interessados em compartilhar, por meio de um mapa, todo o seu conhecimento local no tocante à dados geográficos, como ruas e pontos de interesse (POI).

Serviços como o TripAdvisor ${ }^{2}$, Flickr ${ }^{3}$, Twitter e Panoramio $^{4}$ são considerados exemplos de VGI, entretanto, Stefanidis et al. [14] defendem que a informação disseminada nas mídias sociais é uma variante da VGI, uma vez que ela não é informação geográfica por si, como se vê no OSM. Nas redes sociais, a informação geográfica pode estar relacionada a diferentes contextos sociais. Por esse motivo, dados das redes sociais tendem a ser categorizados como AGI em vez de VGI, pelo fato destes dados irem além da geografia, representando comportamentos dos indivíduos no contexto geográfico.

Dentro do contexto de AGI, encontram-se as Redes Sociais Baseadas em Localização (do inglês, LBSN - Location Based Social Networks), que surgiram da combinação entre redes sociais e os serviços baseados em localização (do inglês, LBS). LBSNs são estruturas sociais compostas por indivíduos, conectados pela interdependência derivada de suas localizações no mundo físico e seu conteúdo multimídia, como fotos, textos e vídeos, associados a uma localização [17]. Tal interdependência estabelece uma relação entre estes indivíduos, na medida que, ao mesmo tempo que eles podem causar efeitos positivos e/ou negativos para toda a sociedade, eles também podem ser influenciados pelos demais indivíduos. Assim, as LBSNs preenchem uma lacuna entre o comportamento dos usuários no mundo digital e físico [3], aproveitando-se dos recursos oferecidos por um SIG.

Exemplos de LBSN incluem o Foursquare ${ }^{5}$, que reúne experiências de pessoas no domínio de entretenimento; o

\footnotetext{
${ }^{2}$ http://www.tripadvisor.com/

${ }^{3}$ https://www.flickr.com/

${ }^{4}$ http://www.panoramio.com/

${ }^{5}$ https://foursquare.com/
} 
Waze $^{6}$, que reúne informação de tráfego e auxilia na navegação dentro das grandes cidades; o MapMyTracks ${ }^{7}$, que monitora atividades físicas utilizando GPS e questões relacionadas à boa condição física dos indivíduos; e ambientes como o Crowd4City [7] e o FixMyStreet [16], que são voltados à queixas da população no domínio das cidades inteligentes. As LBSNs no domínio das cidades inteligentes têm desempenhado um importante papel no monitoramento das cidades, sob diversos aspectos relacionados à qualidade de vida, como melhoria dos serviços públicos, combate à doenças infectocontagiosas e combate à violência. No tocante às LBSNs relacionadas ao monitoramento e combate à doenças, diversas iniciativas têm sido propostas.

Eisen and Lozano-Fuentes [6] defendem como o uso de um SIG pode ser de grande ajuda para facilitar a visualização, análise e o controle do mosquito Aedes aegypti e dos casos de dengue. O trabalho avaliou diferentes formas de modelagem espacial e espaço-temporal, como também formas de mapear dados sobre a dengue, levando em consideração dados epidemiológicos e dados sobre os mosquitos vetores de dengue coletados por fontes oficias. A análise apresentada reforça como o uso de um SIG seria uma fonte de ajuda no controle à dengue, já que com essa tecnologia, seria possível gerar mapas de áreas de risco de exposição ao vírus da dengue e classificações de áreas prioritárias ao combate do mosquito, ajudando assim a população e os órgãos responsáveis à combatê-lo.

Reddy et al. [11] desenvolveram uma aplicação para a plataforma Android, onde usuários que não tenham fácil acesso a postos de saúde possam informar seus sintomas. Caso os sintomas informados indiquem uma suspeita de dengue, o usuário é indicado para ir ao médico. Sendo confirmado pelo médico que o mesmo está com dengue, sua localização capturada pelo dispositivo é então compartilhada em um mapa que monitora a origem de pessoas doentes. As informações do mapeamento de casos confirmados permanecem disponíveis para visualização por um período de vinte dias, garantindo a cobertura de todo o período de incubação da Dengue e evitando que o mapa fique poluído com informações desatualizadas. Um estudo semelhante é apresentado por De Mendonça et al. [4], que utiliza dados abertos da Secretaria de Saúde do Mato Grosso. Mesmo sendo boas ferramentas, remediar é muito mais caro que prevenir. Neste sentido, nossa proposta busca agir sobretudo nos focos que possam encadear novos casos de pessoas com as doenças, antes mesmo que as pessoas venham a adoecer e relatar seus sintomas.

Antunes et al. [1] desenvolveram o e-monitor, um sistema Web que visa ajudar na vigilância de epidemias da dengue. O sistema monitora fontes de informação como sites de noticias, redes sociais, fontes oficiais e fontes de produção científica para informações sobre a dengue, reunindo e exibindo as informações coletadas. O trabalho relata uma análise com postagens do Twitter (tweets) contendo a palavra "dengue" publicados na cidade do Rio de Janeiro, traçando perfis dos usuários que mais falam sobre a doença no Twitter e descobrindo uma relação temporal entre a quantidade de tweets e os períodos de epidemia da doença. Os resultados apresentados reforçam a utilidade do Twitter como uma fonte complementar na vigilância epidemiológica. Entretanto, o

\footnotetext{
${ }^{6}$ https://www.waze.com/

${ }^{7}$ http://www.mapmytracks.com/
}

e-monitor falha em usabilidade, já que apesar da ferramenta reunir notícias sobre a dengue no estado do Rio de Janeiro, não existe uma forma de visualizar as informações por localidades dentro do município, dificultando o monitoramento de possíveis áreas propícias à epidemias de dengue. O DeuZikaChico, por outro lado, é um SIG que possibilita uma visualização dinâmica dos dados distribuídos espacialmente.

Signorini et al. [13] usa o Twitter para fazer uma análise de sentimento em relação à gripe suína e para acompanhar e medir a atividade da doença, mostrando que o Twitter pode sim ser usado para medir o interesse e preocupação do público em relação a eventos envolvendo a saúde. Coletando os tweets utilizando um conjunto pré-definido de palavraschave (como flu, swine e influenza), foi gerado um mapa com os 500 tweets mais recentes, produzindo uma visão em tempo real do público relacionado à gripe no contexto geográfico. Para determinar a contribuição de cada palavra-chave relacionada à gripe, foi criado um modelo de dados usando o Support Vector Regression, uma instância do algoritmo SVM (Support Vector Machine). Os resultados reforçam a utilidade do Twitter na análise de doenças, permitindo estimar, em tempo real, a atividade de doenças de forma mais rápida que a convencional.

Gomide et al. [8] utilizou o Twitter para fazer uma análise de sentimento em relação à Dengue no Brasil, por meio de 4 dimensões: volume, localização, tempo e percepção pública. O autor cruzou os dados adquiridos por meio da rede social com dados oficiais do governo brasileiro para propor, implementar e avaliar um sistema de vigilância ativa contra a dengue. Contudo, o modelo proposto apresenta certo grau de imprecisão, pois utiliza apenas os dados oficiais coletados pelo Ministério da Saúde entre os anos de 2007 e 2010, e todos os tweets que continham a palavra "dengue" coletados entre 2006 e 2009 , e entre 2010 e 2011. Não há um monitoramento em tempo real nem dos últimos anos, além do foco exclusivamente na Dengue, não abordando outras doenças que recentemente tem merecido semelhante atenção, como Zika e Chikungunya, também causadas pelo mosquito Aedes aegypti.

O levantamento do estado da arte deixa claro a falta de soluções que utilizem os recursos de um SIG combinado com a interatividade de uma LBSN e o aproveitamento de dados de redes sociais para um monitoramento em tempo real de epidemias como a da Dengue, Zika e Chikungunya. O DeuZikaChico se destaca principalmente por explorar a AGI obtida nas redes sociais e fornecida diretamente à aplicação por meio de seus usuários, possibilitando o monitoramento e mapeamento de áreas de maior incidência, assim como áreas com maior esforço das autoridades locais. Também, nossa proposta provê o uso de plataformas móveis dando mobilidade e agilidade aos informantes de focos de mosquito, tanto os agentes de saúde como a população em geral.

\section{DEUZIKACHICO: UM AMBIENTE PARA MONITORAMENTO DA DENGUE, ZIKA E CHIKUNGUNYA}

O arcabouço proposto tem como objetivo propiciar um ambiente em que a população participe mais ativamente no monitoramento e combate ao mosquito Aedes aegypti, oferecendo esta interação por meio de um SIG web e de uma aplicação para dispositivos móveis. Esta seção tem como objetivo apresentar o arcabouço desenvolvido, suas caracte- 


\section{DeuZikaChico}

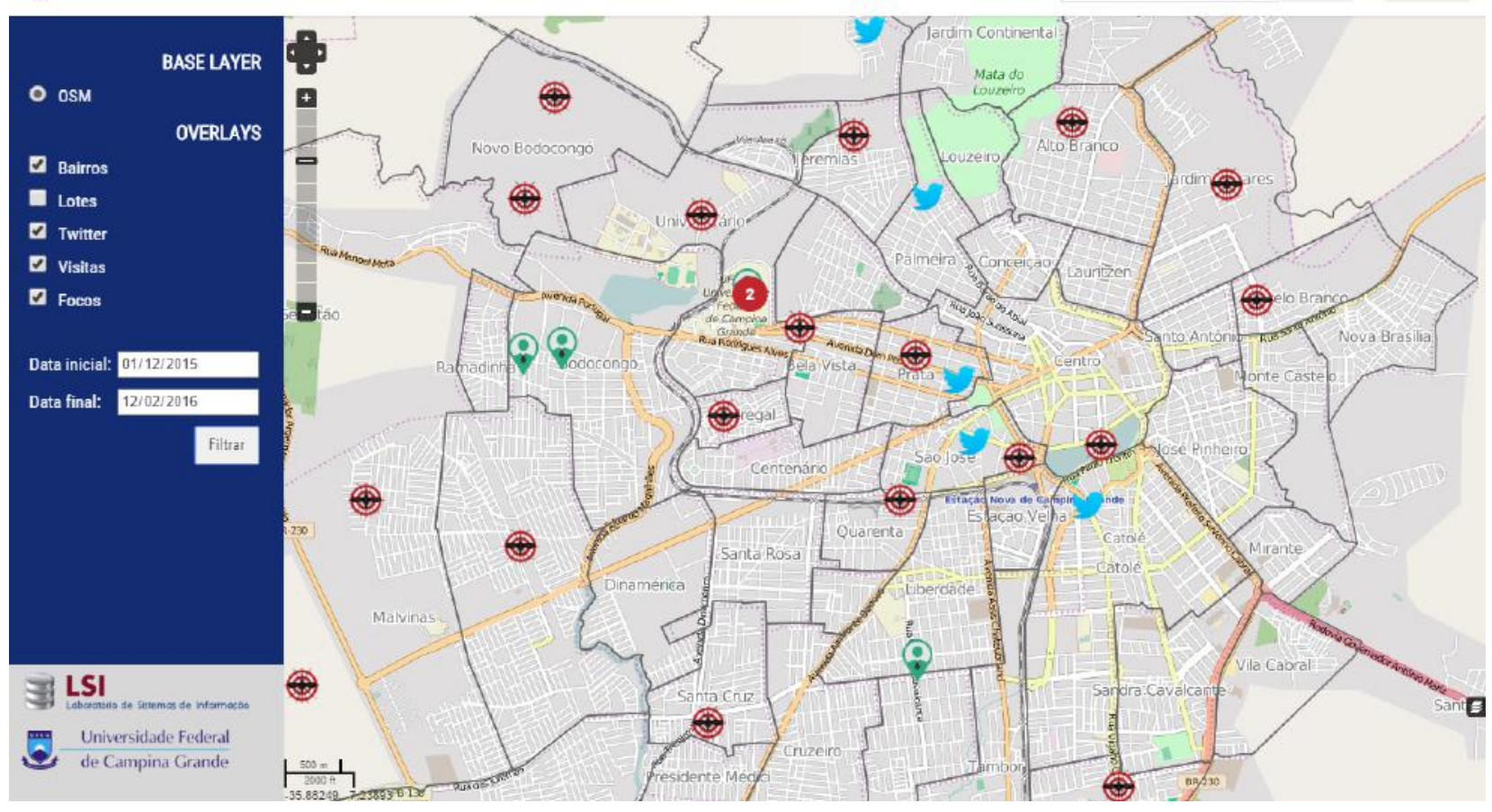

Figura 1: Tela Principal do DeuZikaChico versão web

rísticas, arquitetura e o mecanismo utilizado para extração de dados do Twitter.

O DeuZikaChico foi desenvolvido para tornar eficiente e eficaz as ações de monitoramento e combate ao mosquito, sendo de grande utilidade tanto para a população em geral como para as autoridades de saúde do governo nas diversas esferas: municipal, estadual e federal. A tela principal do SIG é ilustrada na Figura 1.

A Figura 1 representa a tela principal da aplicação onde é disponibilizado para o usuário diferentes camadas para que ele possa selecionar o que deseja visualizar. A camada base do mapa é fornecida pelo OpenStreetMap (OSM). O usuário pode sobrepor cinco camadas no mapa: Bairros, Lotes, Twitter, Visitas e Focos. Na camada "Bairros" são exibidos os limites de cada bairro do município, delimitações na cor cinza. Na camada "Lotes" são exibidos todos os lotes da cidade. Na camada "Twitter" são exibidos os dados coletados no Twitter referentes à localidade exibida no mapa, representados com o ícone de um pássaro azul. A camada "Visitas" exibe os dados sobre as visitas dos agentes de saúde informadas pelos usuários, representadas como o ícone de marcadores verdes. Por fim, a camada "Focos" exibe os dados sobre os possíveis focos do mosquito, representados pelo intuitivo ícone de um mosquito sobre um alvo. Os ícones representativos das camadas são substituídos por clusters de acordo com o quantitativo de dados em uma mesma área $\mathrm{e}$ o nível de zoom que o usuário aplica sobre o mapa. As cores dos clusters representam uma abstração dos ícones que distinguem as camadas. A tela principal conta ainda com um filtro temporal, onde o usuário pode informar um intervalo de tempo para visualização de dados históricos; e com um campo de busca no canto superior, para busca de endereços no mapa.

São funcionalidades do DeuZikaChico:

Denunciar possíveis focos: possibilita descrever focos do mosquito (recipientes com água parada, lixo acumulado, casa abandonada, etc.) em detalhes, incluindo a localização geográfica e uma fotografia georreferenciada do local, permitindo que as autoridades locais tomem as devidas providências;

Informar locais visitados por autoridades de saúde: permite descrever com detalhes as ações tomadas em uma localização geográfica específica, permitindo o mapeamento das áreas de um município em que as autoridades já tomaram alguma providência no combate ao mosquito, por lote, bairro e município;

Visualizar tweets relacionados às doenças causadas pelo Aedes aegypti: possibilita a visualização de tweets geolocalizados que indiquem possíveis focos ou casos de pessoas que adoeceram, enriquecendo o mapeamento das doenças. É possível clicar no link do tweet e ser redirecionado para a mensagem original, onde é possível acompanhar sua repercussão;

Filtrar possíveis focos, visitas por autoridades e tweets de acordo com a data: permite a visualização dos pontos de interesse (focos, visitas e/ou tweets) de acordo com o intervalo de tempo desejado, indicando a data inicial e a data final. Esse recurso possibilita uma visualização do histórico de registros;

Buscar endereço: permite a centralização do mapa para o endereço desejado. A aplicação pode utilizar os recur- 


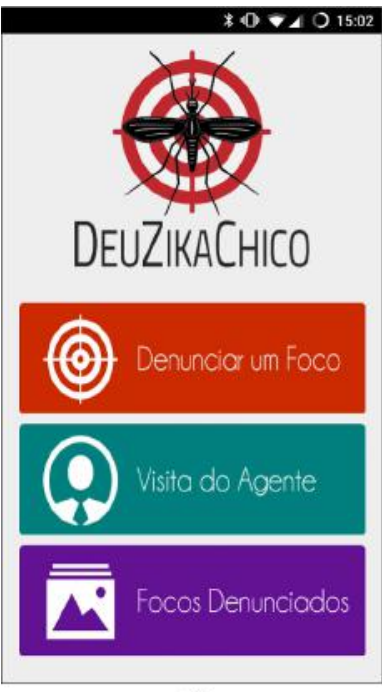

a)

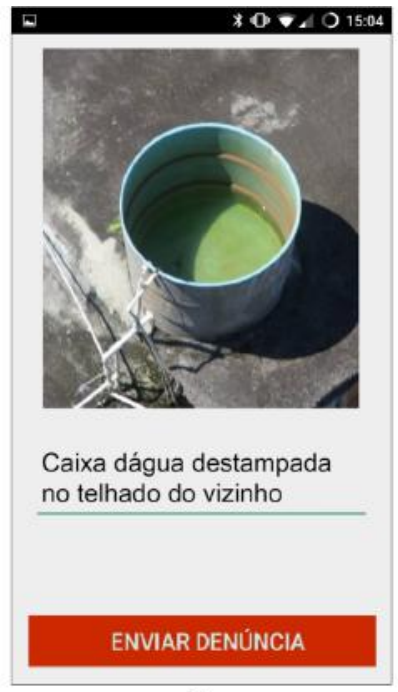

b)

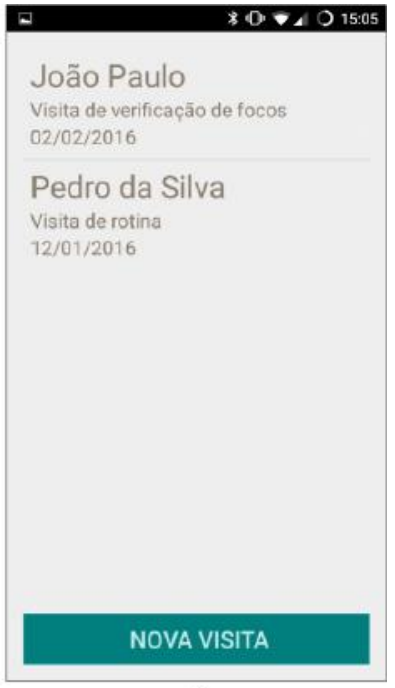

c)

Figura 2: Principais telas do DeuZikaChico versão mobile

sos de identificação de localização do navegador web ou do dispositivo móvel para automaticamente redirecionar o usuário para seu local atual.

Além do SIG on-line, o DeuZikaChico conta com uma versão mobile, que pode ser utilizada em smartphones e tablets, dando mais praticidade aos usuários de dispositivos móveis, que podem enviar suas denúncias à medida que estas forem identificadas in-loco. Os agentes de saúde também podem utilizar-se desta versão para registrar suas visitas à medida que estas forem realizadas. A versão mobile foi desenvolvida voltada para a praticidade dos usuários em postar suas denúncias e visitas, ficando a visualização dos dados para a versão web, que também permite o registro de visitas e denúncias. A Figura 2 ilustra as principais telas do DeuZikaChico mobile.

A Figura 2a representa a tela inicial da versão mobile que consiste de três botões. O usuário pode denunciar um foco clicando no botão "Denunciar um foco", que o redirecionará para o aplicativo de câmera do dispositivo móvel para capturar uma imagem relacionada à denúncia e em seguida informar a descrição do foco, como ilustrado na Figura 2b. A coordenada geográfica do local da denúncia é capturada automaticamente utilizando os recursos de localização do dispositivo. O botão "Visita do Agente" possibilita o cadastro de visitas de agentes. A Figura 2c exibe uma lista com as visitas já cadastradas pelo usuário e a opção para cadastrar uma nova a partir de um formulário. Por fim, o botão "Focos denunciados" possibilita visualizar o histórico de todos os focos denunciados pelo usuário.

\subsection{Arquitetura}

Para o desenvolvimento da arquitetura do DeuZikaChico, foi adotado o modelo em três camadas, que consiste de: camada de apresentação; camada de negócio; e camada de dados. A arquitetura do ambiente é ilustrada na Figura 3.

A camada de apresentação contém os elementos exibidos ao usuário final utilizados para a interação com o ambiente. As informações apresentadas são provenientes dos sensores humanos que as inserem diretamente por meio da própria aplicação ou a partir de postagens na rede social Twitter. A versão web foi desenvolvida utilizando o OpenLayers $^{8}$, uma ferramenta de software livre para visualização de mapas muito utilizada em SIGs na web, que oferece recursos básicos de navegação como manipulação de camadas, pan e zoom. A versão mobile, por outro lado, utiliza os recursos padrão de aplicativos do sistema operacional Android.

A camada de negócios é responsável pelo processamento das operações requisitadas pelos usuários e contém toda a lógica de negócio do sistema desenvolvido utilizando a linguagem de programação PHP. Nesta camada, há um motor de busca (crawler) que foi desenvolvido para ficar em execução localizando tweets georeferenciados que estejam associados ao mosquito Aedes aegypti ou às doenças transmissíveis pelo mosquito. O crawler será detalhado mais adiante. Foi utilizado como servidor de mapa da aplicação o Geoserver ${ }^{9}$. O mesmo foi escolhido por ser um software livre que utiliza padrões abertos definidos pela Open Geospatial Consortium (OGC) para o acesso a dados espaciais, como o Web Map Service (WMS) e o Web Feature Service (WFS). A partir desses padrões de acesso, o Geoserver estabelece uma conexão direta com o OpenLayers na camada de visualização, abstraindo a comunicação com a camada de dados.

Por fim, a camada de dados é utilizada para armazenar todos os dados gerados e processados pela camada de negócio e que serão visualizados na camada de apresentação. Há três repositórios de dados: tweets provenientes do motor de busca; arquivos multimídia compartilhados pelos usuários na aplicação; e uma instância do SGBD PostgreSQL para armazenar os dados de cadastro e também os dados geográficos através de sua extensão PostGIS.

\subsection{Crawler de Tweets}

$\mathrm{O}$ crawler foi desenvolvido em Java, utilizando a $\mathrm{API}^{10}$

\footnotetext{
${ }^{8}$ http://openlayers.org/

${ }^{9}$ http://geoserver.org/

${ }^{10}$ https://dev.twitter.com/streaming/
} 


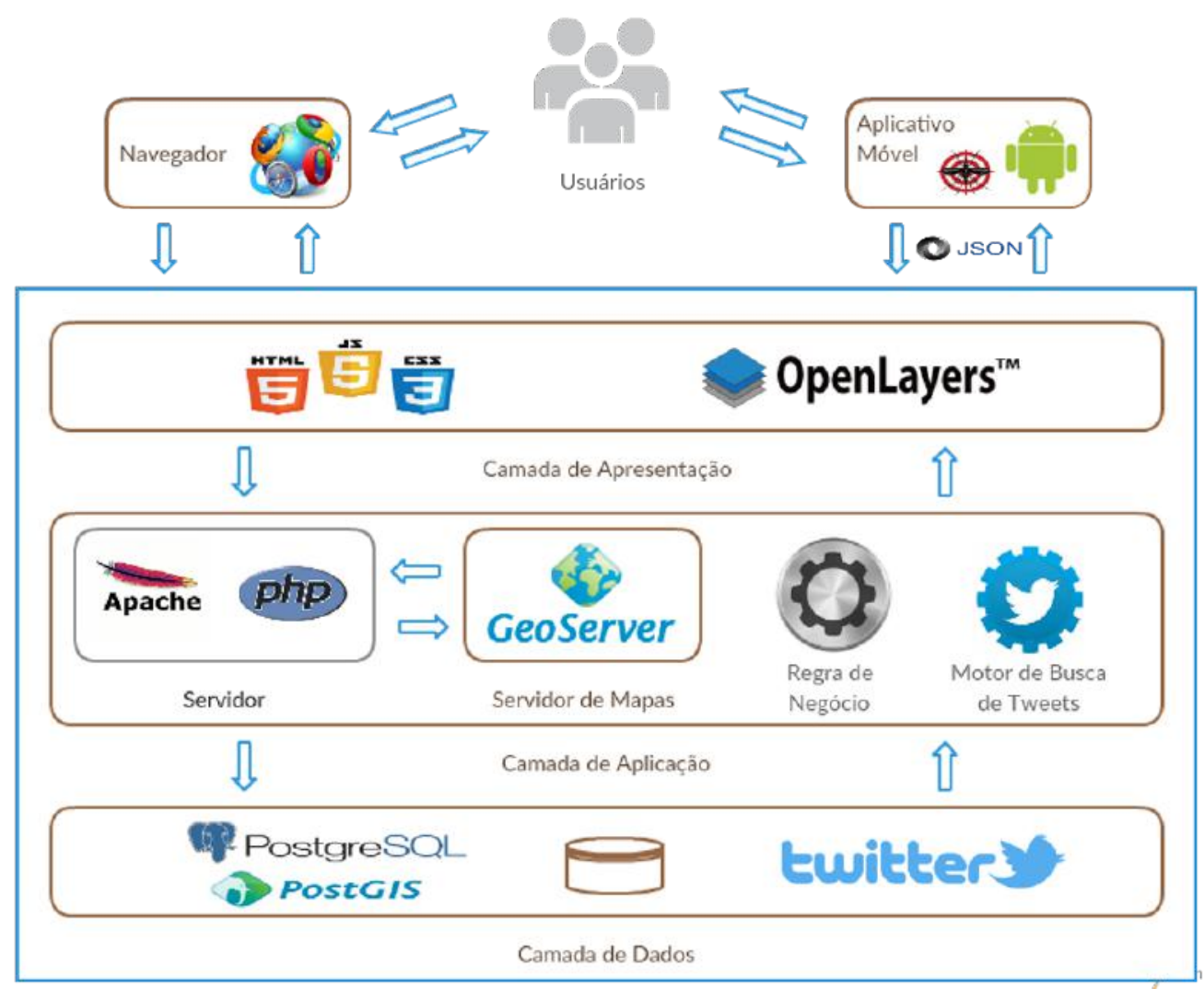

Figura 3: Arquitetura do ambiente proposto

disponibilizada pelo Twitter para monitoramento de Tweets em tempo real. A filtragem de tweets relacionados com o mosquito Aedes aegypti é realizada em três fases:

- Na primeira fase, utiliza-se a API de streaming do Twitter para receber os tweets postados em um determinado município. Tal restrição espacial garante que todos os tweets filtrados serão georeferenciados e poderão ser plotados no mapa do DeuZikaChico. Tweets não georeferenciados exigiriam geoparsing e, portanto, serão explorados em trabalho futuro;

- Na segunda fase, aplica-se um filtro textual sobre o conjunto de tweets recebidos na primeira fase. Tal filtro identifica tweets com mensagens contendo termos como "dengue", "aedes", "água parada", "zika", "foco", dentre outros;

- Na terceira e última fase, aplica-se um outro filtro textual para remover mensagens que contenham palavras ou expressões de cunho ofensivo. Este filtro também tenta remover mensagens que possam ser consideradas spam, como publicidade.

Os tweets filtrados recebidos pelo crawler são armazenados em arquivos no formato JSON (JavaScript Object Notation), padrão da API do Twitter, que armazena não apenas a mensagem como todos os metadados (ids, geolocalização, URLs mencionadas, dados do usuário que postou o tweet, etc.).

\section{DEUZIKACHICO NA PRÁTICA: UM ES- TUDO DE CASO}

Um estudo de caso com o objetivo de avaliar o DeuZikaChico foi realizado considerando o Estado da Paraíba, enfatizando a cidade de Campina Grande, segunda maior cidade desse Estado, que ganhou destaque na mídia internacional devido aos estudos iniciais de associação entre o Zika vírus e os casos de Microcefalia em bebês [2]. O objetivo do estudo de caso é avaliar os potenciais da ferramenta, através da exploração da AGI proveniente tanto de redes sociais, através de coleta de tweets, quanto de informações oriundas da própria aplicação, seja de um sistema Web ou de um aplicativo para dispositivo móvel.

A avaliação foi realizada durante os meses de dezembro de 2015 e janeiro de 2016 , no qual foi disponibilizado aplicativo no Google Play ${ }^{11}$, para instalação em dispositivos móveis, e o endereço eletrônico ${ }^{12}$ do aplicativo web. A divulgação do aplicativo foi realizada pelos autores por meio de redes sociais como o Facebook. Durante este período, o motor de busca de tweets foi habilitado para localizar tweets geolocalizados em que suas coordenadas estivessem contidas na área geográfica correspondente ao Estado da Paraíba, e cujo conteúdo estivesse associado com o mosquito Aedes aegypti,

\footnotetext{
$\overline{{ }^{11} \text { https://play.google.com/store/apps/details?id=br.edu.ufc }}$ g.lsi.deuzicachico

${ }^{12} \mathrm{http}: / /$ www.deuzikachico.com/
} 


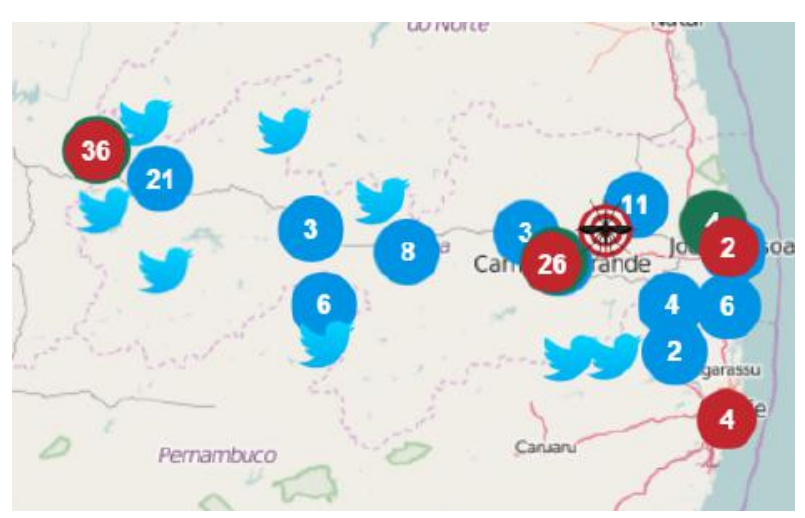

Figura 4: Dados de denúncias de focos, visitas realizadas e tweets (Paraíba)

conforme já mencionado. Para não utilizar dados apenas dos tweets coletados, cerca de 10 voluntários foram convidados a utilizar a aplicação, seja do dispositivo móvel ou do sistema Web, com o propósito de realizar denúncias relacionadas a focos do mosquito e envio de fotos georeferenciadas para exploração da ferramenta SIG.

Os voluntários coletaram dados em diferentes localizações para permitir uma exploração espacial da aplicação. Simulando a ação do poder público na aplicação, também foi solicitado que alguns voluntários fizessem a inserção de dados sobre prováveis visitas de agentes de saúde pública. É importante mencionar, que não necessariamente precisa-se do poder público para cadastrar dados de uma visita de um agente, uma vez que o próprio cidadão, ao ter sua casa visitada, poderá fazer este registro. As Figuras 4 e 5 mostram os focos e visitas cadastrados, juntamente com os tweets, considerando todo o Estado da Paraíba (Figura 4) e, com mais detalhes, na cidade de Campina Grande (Figura 5). Quando o nível de zoom é menor, como na Figura 4, os marcadores são substituídos por clusters contendo o número de marcadores agrupados na região geográfica representada. Com um nível de zoom maior, que permite ver melhor bairros e ruas de um município, por exemplo, os clusters tendem a ser substituídos por marcadores espaciais a medida que estes se distanciam geograficamente no nível de visualização utilizado, a exemplo da Figura 5.

Com os dados coletados, foi possível identificar as localizações que o poder público poderia intensificar nas fiscalizações e nas ações socio-educacionais com o objetivo de prevenir a proliferação do mosquito. Além do mais, a avaliação do trabalho dos agentes de saúde através das ações empreendidas poderia ser mensurada, verificando, mesmo depois de visitas, se há reincidência de denúncias de focos em locais já visitados, através de uma análise temporal e espacial. Esta análise temporal e espacial, pode ser realizada no sistema web, por exemplo, através dos filtros temporais, criando assim um histórico sobre os focos, visitas e tweets. $\mathrm{O}$ arcabouço proposto permite uma sociedade mais ativa no combate do mosquito, uma vez que ela pode realizar as denúncias e acompanhar as ações do poder público.

Também é disponibilizado no sistema diferentes camadas nas quais o usuário pode escolher o que exatamente ele está procurando. Por exemplo, se um usuário quer ter uma visão mais limpa onde estão vindo as informações coletadas no

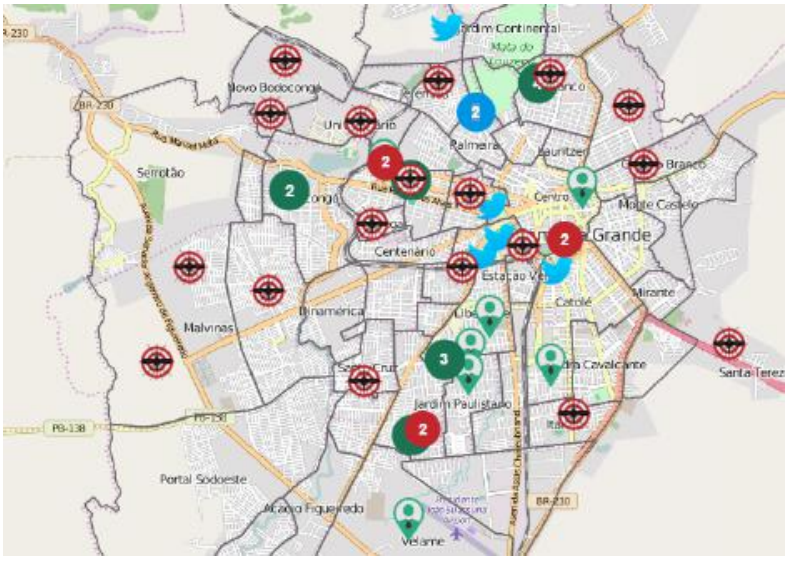

Figura 5: Dados de denúncias de focos, visitas realizadas e tweets (Campina Grande)

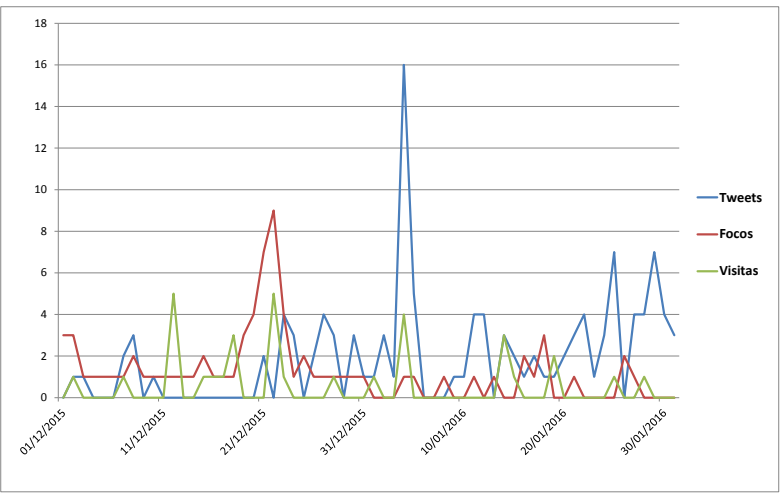

Figura 6: Distribuição temporal dos registros coletados

twitter, basta desmarcar as camadas que não lhe interessar. Uma boa aplicação das camadas é quando deseja comparar os focos denunciados com os locais visitados, selecionando apenas as duas camadas desejadas. O mesmo princípio pode ser realizado quando se deseja destacar os bairros com mais denúncias de focos ou mais vistoriados pelos agentes públicos.

Com relação aos dados coletados durante o período do estudo, a Figura 6 ilustra a quantidade de registros coletados, por fonte de dados, após a disponibilização da ferramenta.

Como pode ser visto, o número de denúncias de possíveis focos do mosquito foi maior durante o mês de dezembro de 2015, mês no qual a ferramenta foi disponibilizada. O número de dados referentes às visitas do agente também foram maior durante o mês de dezembro de 2015. Já a quantidade de tweets coletados no estado da Paraíba foi maior no mês de janeiro de 2016, com uma concentração maior entre os dias $03 / 01$ e $05 / 01$, data a qual foi divulgada uma notícia ${ }^{13}$ de que o Governo do Estado distribuiu kits ao exército para ajudar no combate ao mosquito Aedes aegypti.

\footnotetext{
${ }^{13}$ http://www.clickpolitica.com.br/noticia/1897/exercitobrasileiro-entra-na-luta-contra-mosquito-aedes-aegypticonfira-aqui.html
} 
O uso da ferramenta permite um acompanhamento em tempo real do poder público, uma vez que pode realizar as devidas intervenções nas regiões mais necessárias, conforme denúncias realizadas, inclusive nas mídias sociais. Além disso, o cruzamento de informações de endereços de pessoas infectadas com a relação de denúncias e visitas realizadas pode auxiliar de forma efetiva no combate ao mosquito.

\section{CONCLUSÃO E TRABALHOS FUTUROS}

Este artigo apresentou o DeuZikaChico, um arcabouço tecnológico composto de sistemas Web e mobile para o auxílio da população e dos agentes públicos no combate e monitoramento de epidemias, como a de doenças causadas pelo mosquito Aedes aegypti. A solução proposta é composta por duas aplicações: um SIG web e um aplicativo para dispositivos móveis. O principal objetivo da proposta é reunir, em tempo real e de forma intuitiva para a população, informações oficiais de agentes públicos e Informação Geográfica Ambiental (AGI) fornecida por sensores humanos tanto diretamente, por meio das aplicações desenvolvidas, como através de postagens no Twitter.

Um estudo foi realizado no estado da Paraíba, no Brasil, de modo a avaliar o potencial da solução proposta no combate às doenças causadas pelo mosquito Aedes aegypti, que tem causado grande preocupação da população. Os resultados demonstram o potencial da AGI para ações imediatas e eficientes dos agentes de saúde, que identificaram municípios com grande quantidade de focos do mosquito transmissor e puderam direcionar suas ações de modo a visitar os locais identificados e tomar as providências cabíveis.

Um trabalho futuro focará no desenvolvimento do crawler de tweets, de modo a reduzir o número de falsos positivos e mensagens de cunho publicitário. Outro trabalho futuro envolverá um estudo de caso de médio prazo com um conjunto de agentes públicos atuando em um município específico, de modo a identificar pontos que possam ser evoluídos na solução proposta. Também, pretende-se aplicar técnicas de análise de sentimento para descobrir a polaridade dos tweets. Por fim, se pretende integrar a ferramenta com dados públicos de saúde das fontes oficiais, possibilitando correlacionar essas informações e a AGI disponível no DeuZikaChico.

\section{REFERÊNCIAS}

[1] M. N. Antunes, C. H. d. Silva, M. C. S. Guimarães, and M. H. L. Rabaco. Monitoramento de informação em mídias sociais: o e-Monitor Dengue. Transinformação, 26:9-18, 2014.

[2] G. Calvet, R. S. Aguiar, A. S. O. Melo, S. A. Sampaio, I. de Filippis, A. Fabri, E. S. M. Araujo, P. C. de Sequeira, M. C. L. de Mendonça, L. de Oliveira, D. A. Tschoeke, C. G. Schrago, F. L. Thompson, P. Brasil, F. B. dos Santos, R. M. R. Nogueira, A. Tanuri, and A. M. B. de Filippis. Detection and sequencing of Zika virus from amniotic fluid of fetuses with microcephaly in Brazil: a case study. The Lancet Infectious Diseases, pages 1-8, 2016.

[3] J. Cranshaw, E. Toch, J. Hong, A. Kittur, and N. Sadeh. Bridging the Gap Between Physical Location and Online Social Networks. In Proceedings of the 12th ACM International Conference on Ubiquitous Computing, UbiComp '10, pages 119-128, New York, NY, USA, 2010. ACM.
[4] P. G. A. de Mendonça, C. Maciel, and J. V. Filho Visualizing aedes aegypti infestation in urban areas: A case study on open government data mashups. In Proceedings of the 15th Annual International Conference on Digital Government Research, dg.o '14, pages 186-191, New York, NY, USA, 2014. ACM.

[5] H. B. M. Diniz, E. C. G. F. Silva, and K. S. da Gama. A reference architecture for a crowdsensing platform in smart cities. In Proceedings of the Annual Conference on Brazilian Symposium on Information Systems, SBSI 2015, pages 13:87-13:94, Porto Alegre, Brazil, 2015. Brazilian Computer Society.

[6] L. Eisen and S. Lozano-Fuentes. Use of mapping and spatial and space-time modeling approaches in operational control of Aedes aegypti and dengue. PLoS Negl Trop Dis, 3(4):e411, 2009.

[7] A. G. R. Falcão, C. d. S. Baptista, and L. d. Menezes. Crowd4City: utilizando sensores humanos como fonte de dados em cidades inteligentes. In Proceedings of the Annual Conference on Brazilian Symposium on Information Systems, SBSI 2012, pages 144-149, São Paulo, Brazil, 2012. Brazilian Computer Society.

[8] J. Gomide, A. Veloso, W. Meira, Jr., V. Almeida, F. Benevenuto, F. Ferraz, and M. Teixeira. Dengue Surveillance Based on a Computational Model of Spatio-temporal Locality of Twitter. In Proceedings of the 3rd International Web Science Conference, WebSci '11, pages 3:1-3:8, New York, NY, USA, 2011. ACM.

[9] M. F. Goodchild. Citizens as voluntary sensors: spatial data infrastructure in the world of web 2.0. International Journal of Spatial Data Infrastructures Research, 2:24-32, 2007.

[10] M. Haklay and P. Weber. OpenStreetMap: User-Generated Street Maps. IEEE Pervasive Computing, 7(4):12-18, 2008.

[11] E. Reddy, S. Kumar, N. Rollings, and R. Chandra. Mobile application for dengue fever monitoring and tracking via GPS: case study for fiji. CoRR, abs/1503.00814, 2015 .

[12] Secretaria de Vigilância em Saúde, Ministério da Saúde, Brasil. Boletim Epidemiológico. 47(3), 2016. Disponível em: http://portalsaude.saude.gov.br/images/pdf/2016/ janeiro/15/svs2016-be003-dengue-se52.pdf.

[13] A. Signorini, A. M. Segre, and P. M. Polgreen. The Use of Twitter to Track Levels of Disease Activity and Public Concern in the U.S. during the Influenza A H1N1 Pandemic. PLoS ONE, 6(5):e19467, 2011.

[14] A. Stefanidis, A. Crooks, and J. Radzikowski. Harvesting ambient geospatial information from social media feeds. GeoJournal, 78(2):319-338, 2013.

[15] J. Surowiecki. The Wisdom of Crowds. Knopf Doubleday Publishing Group, 2005.

[16] N. Walravens. Validating a Business Model Framework for Smart City Services: The Case of FixMyStreet. In Proceedings of the 27th International Conference on Advanced Information Networking and Applications Workshops (WAINA), pages 1355-1360, 2013.

[17] Y. Zheng, L. Capra, O. Wolfson, and H. Yang. Urban Computing: Concepts, Methodologies, and Applications. ACM Trans. Intell. Syst. Technol, $5(3): 1-55,2014$ 